Voix et Images

volxetimages

\title{
Ramos et Ringuet : le roman entre le silence et l'histoire
}

\section{Javier García Méndez}

Volume 12, numéro 1 (34), automne 1986

Québec-Amérique latine

URI : https://id.erudit.org/iderudit/200606ar

DOI : https://doi.org/10.7202/200606ar

Aller au sommaire du numéro

Éditeur(s)

Université du Québec à Montréal

ISSN

0318-9201 (imprimé)

1705-933X (numérique)

Découvrir la revue

Citer cet article

Méndez, J. G. (1986). Ramos et Ringuet : le roman entre le silence et l'histoire.

Voix et Images, 12(1), 55-66. https://doi.org/10.7202/200606ar d'utilisation que vous pouvez consulter en ligne.

https://apropos.erudit.org/fr/usagers/politique-dutilisation/ 


\title{
Ramos et Ringuet: le roman entre le silence et l'histoire
}

\author{
par Javier García Méndez, Université du Québec à Montréal
}

\author{
À la mémoire de André Belleau \\ - Bon, bon, c'est correct; moé c'que j'disais \\ c'était comme manière de parler...
}

Euchariste Moisan

Je fouille quand tu parles

on ne s'arrête plus quand on écoute

Henri Meschonnic

L'année 1938 est pour l'historien une prodigieuse collection d'événements invitant à des mises en rapport aussi aventureuses qu'infinies: lois antisémites en Italie, victoire du Front populaire au Chili, présence japonaise à Canton, conférence panaméricaine à Lima, révoltes contre l'administration française en Tunisie, nationalisation du pétrole au Mexique, occupation allemande de l'Autriche, accords de Munich, retrait des Brigades internationales de l'Espagne. Pour ce qui est des faits esthétiques, la même année n'est pas moins bigarrée: la Nausée de J.-P. Sartre, Superman de Siegel et Shuster, Quai des brumes de Marcel Carné, la Figure inclinée de Henry Moore, Blanche Neige de Walt Disney, Billy the Kid de Aaron Copland, Macbeth de Orson Wells. Aussi, les deux romans que le présent texte veut saisir en un seul mouvement de pensée: Trente arpents de Ringuet, Vidas secas de Graciliano Ramos.

La tâche ne comporte pas moins d'embûches que celle de l'historien, surtout si, en l'accomplissant, on veut tenir compte du fait que ces romans sont aussi des événements historiques. Les mettre en rapport peut être en effet aussi hasardeux qu'établir des liens entre la campagne anti-trust aux États-Unis, la bataille de l'Ebre et l'ultimatum allemand à Prague. Pas autant, en vérité. Outre le fait d'avoir été publiées la même année, Vidas secas et Trente arpents sont des œuvres littéraires appartenant au même genre, écrites de surcroît dans des lieux américains façonnés par la colonisation européenne et ayant reçu de l'Europe la forme romanesque. Toutefois, la distance sociale et culturelle entre le Québec et le Brésil - produit de deux processus historiques radicalement différents - est incommensurable, comme l'est d'ailleurs, à cause de la même diversité historique, celle qui, en 1938, sépare le roman brésilien - et, plus largement, le roman latino-américain - de la fiction narrative québécoise.

Il suffit pour trouver une illustration frappante de cette distance, de remonter au milieu du $\mathrm{XIX}^{\mathrm{c}}$ siècle afin de comparer les portraits que deux romans publiés presque en même temps font des habitants de leurs sociétés de référence, ainsi que les idéaux scripturaux qu'ils proposent. Alors que la Terre paternelle (1846), de Patrice Lacombe, suggère de laisser aux vieux pays que la civilisation a gâtés, leurs romans ensanglantés et de peindre l'enfant du sol, tel qu'il est, religieux, honnête, paisible de mours et de caractère, Facundo (1845), de Domingo Faustino Sarmiento, fait un tableau 
brutal et sanglant du gaucho et proclame ce personnage, ainsi que la lutte entre la "barbarie» et la "civilisation", sujets propres à une littérature argentine susceptible d'être reconnue en Europe. La confrontation des deux exemples montre deux conceptions littéraires opposées, l'une tournée vers la contradiction et vers le reste du monde - même si ce dernier est circonscrit à l'Europe -, l'autre chantant le repli sur soi et une harmonie idéale. À quelques années de leur émergence, les rejetons québécois et latino-américains du roman européen se trouvent sur des chemins tout à fait divers. Près d'un siècle plus tard, Trente arpents et Vidas secas sont parties et témoins des différences entre ces chemins.

Des différences, mais aussi des ressemblances. Il s'agit d'abord de deux textes s'inscrivant dans des traditions génériques que les études littéraires des deux extrémités du continent baptisèrent des mêmes noms : roman régionaliste, roman de la terre. En outre, les deux récits travaillent des diégèses que leur similitude structurale permet de résumer avec les mêmes mots: leurs protagonistes, après avoir souffert de nombreux malheurs tant sociaux que naturels, sont contraints de quitter la campagne pour la ville. Troisièmement, Vidas secas et Trente arpents manifestent un intérêt marqué pour la réalité sociale immédiate, en ce qu'ils sont bâtis autour de problèmes aigus de leur société de référence. Des problèmes ayant sollicité, au même moment, les discours politique, journalistique et scientifique: pour le Nord-est du Brésil, la sécheresse et la dépossession sociale des paysans, l'exploitation à laquelle les propriétaires terriens soumettent leurs salariés, les abus de pouvoir des autorités militaires et policières; pour le Québec, le système traditionnel de legs de la terre, les difficultés des communautés rurales à se mettre au diapason de l'âge industriel, l'attrait exercé par les villes modernes sur lẹ jeunes paysans. D'un côté comme de l'autre, la dureté de la vie rustique, les misères imposées aux travailleurs ruraux par les avatars climatiques, les pratiques par lesquelles les gens instruits tirent profit du manque d'instruction des paysans.

Les rapports qu'on peut établir entre ces deux romans aux plans génétique, thématique ou documentaire sont, on le voit, loin d'être sans intérêt. Mais d'autres relations - autant de ressemblance que de dissemblance paraissent plus riches à explorer: celles qui tiennent à la condition d'objets esthétiques des deux textes, à leur régime de représentation de la réalité et des discours.

\section{Le silence ringuétien ${ }^{1}$}

Ringuet - lit-on dans le Roman à l'imparfait - brosse le décor, la situation d'ensemble, avant de faire agir ou parler ses protagonistes ${ }^{2}$. Essentiellement juste, car elle rend bien compte de l'impression globale que la prose ringuétienne laisse chez le lecteur, la remarque n'est toutefois possible que grâce à une omission. L'omission du début mềme de Trente arpents. Un début que, à cause de l'énonçant et de la forme énonciative, on dirait conçu dans le dessein de décourager une telle caractérisation. Pas de décor au début de ce roman, ni de situation d'ensemble, mais une action, et plus précisément une action verbale: 


\section{- On va commencer betôt les guérêts, m'sieu Branchaud. Mon oncle m'a dit comme ça en partant: "Y faudra labourer le champ en bas de la côte, demain".}

Mais pourquoi s'attarder à ce détail, alors que l'on reconnaît la pertinence de la remarque incidente de Marcotte, alors que l'ensemble de Trente arpents contredit la manière de procéder de son début? Précisément parce qu'il y a contradiction et que l'un des termes de cette contradiction se trouve dans le premier vagissement du texte. Au commencement, une parole d'habitant. Avant d'exécuter le moindre geste descriptif, avant même de laisser voir sa brosse hégémonique, le narrateur fait parler un personnage - le protagoniste - dont le lecteur n'a aucun renseignement. Le choix du procédé énonciatif inaugural dit assez l'intention du romancier de mettre sa parole sous l'égide de la parole paysanne. Mais Trente arpents ne tient pas le pari de son incipit. Ce pari, en fait, ne dure que le temps de la parole d'Euchariste Moisan, la démission advenant avec l'arrivée même de la voix du narrateur à la première page du texte. Une voix qui dit que le désir d'intégrer la parole paysanne se double d'une tendance opposée à la mettre à l'écart, une voix identifiant cette parole comme objet soumis à une dialectique de l'appropriation et du rejet. Les premiers mots du roman que le narrateur prend en charge, immédiatement après les paroles d'Euchariste, sont: Les deux hommes se turent. Cinq mots suffisent à manifester l'impossibilité chez le narrateur de faire chœur avec ses personnages. Cinq mots avec lesquels le narrateur déploie sa voix et, dans le même mouvement, fait taire la voix paysanne. Entre une voix et l'autre, le silence. Ce silence est nécessaire au narrateur - c'est lui, et nul autre, qui le crée. Mais ce silence, il ne pourra le saisir en tant que nécessité à lui, car cela exigerait qu'il prenne conscience de son incapacité à réaliser le projet d'accorder la parole aux paysans. Cependant, pour que soit possible le manque de conscience - condition d'existence du roman - , le narrateur devra faire quelque chose de ce silence essentiel. Il lui faudra tour à tour l'effacer, l'esquiver, le combattre, l'expliquer, bref, le soumettre à des opérations susceptibles de masquer sa véritable nature. De ces opérations, tous les niveaux de composition du texte porteront l'empreinte. À ce point qu'on pourra définir Trente arpents, au plan compositionnel, comme un ensemble de procédés voués à combler son silence initial.

L'échec de la tentative d'intégrer la parole paysanne paraît renforcer la résolution du narrateur, dont le désir premier déterminera ses choix énonciatifs. Il aura systématiquement recours, par exemple, au discours direct des personnages. Mais représentée, la parole paysanne n'est qu'une curiosité syntaxique et lexicale, un lieu de rassemblement d'archaïsmes et de solécismes, une version primitive et maladroite de la parole qui la représente. Elle reste étrangère au discours romanesque qui essaie de la saisir mais qui ne réussit qu'à l'éclairer de l'extérieur. Qui ne réussit, en fait, qu'à la rendre ridicule, cambrée qu'elle est sous le poids des apostrophes et autres marques dont elle est accoutrée. L'échec se reproduit aux innombrables moments où le narrateur fait appel aux bons offices du discours indirect libre, dans le but d'abolir la distance qui le sépare de ses personnages. En fait, la présence souvent excessive du narrateur dans le discours indirect libre montre que celui-ci n'est, chez 
Ringuet, pour l'essentiel, qu'une pure apparence. Son écriture ne garde de cette forme que l'aspect technique, car la voix du narrateur domine (au sens musical, et dans tous les sens) les autres voix: elle les dénature, les assourdit, en efface même complètement les accents. Le discours indirect libre, forme du partage et du rapprochement espacé — non de l'éloignement - , aboutit paradoxalement à exiler les personnages, à les baillonner, à ne laisser place dans le texte qu'à l'omniloquence académicienne du narrateur. Celui-ci traduit "en français» la parole paysanne, la montrant comme issue d'une langue étrangère à celle du roman. La langue campagnarde québécoise ne s'est pas faite texte, elle n'est restée que chose à être observée, traduite et commentée à partir d'une langue autre, celle du romancier et du lecteur implicite. Une langue tellement étrangère aux membres de la société de référence du roman qu'elle les désigne à l'aide du mot paysans, alors qu'eux-mêmes se nomment habitants $^{3}$. Un seul personnage de Trente Arpents parle la langue de son narrateur. Et il n'est pas un habitant québécois mais un paysan français: Albert Chabrol. Personnification du narrataire du roman, Chabrol est aux Moisan ce que les lecteurs de France sont au narrateur. Lorsque les Moisan entretiennent Chabrol de leur propre pittoresque, il ne font qu'imiter ce narrateur qui parle aux Français dans leur propre langue pour leur servir une inépuisable théorie de remarques d'ordre sociologique, ethnographique et linguistique sur la vie rurale québécoise.

Autant que dans le choix des formes énonciatives, l'impossibilité chez le narrateur de saisir la parole paysanne laissera ses traces dans la construction des personnages. Incapable d'accepter le sens du silence dont il a besoin, le maître de la parole du roman en fera un attribut de ses héros. Un attribut qui, d'être purement nominal ${ }^{4}$, exhibe sa condition de pure postiche, d'étoupe de calfatage venant boucher le trou de silence de la première page. Difficilement explicable d'après la réalité même des personnages que le roman construit, le mutisme des Moisan devra avoir sa source dans l'en-dehors du roman. Il trouvera effectivement un appui solide dans un savoir tout fait, que le roman n'aura qu'à citer, selon lequel les paysans sont taciturnes; selon lequel, plutôt, pour respecter l'essentialisme de la proposition, le Paysan est taciturne:

Ils parlaient lentement et peu, à leur accoutumée, étant paysans, donc chiches de paroles 5 .

\section{D'un silence à l'autre}

Ce n'est pas sans une certaine surprise que l'on retrouve dans Vidas secas une insistance sur le laconisme de ses personnages paysans, comparable à celle de Trente arpents. La coïncidence est d'autant plus curieuse qu'elle apparaît, comme on l'a signalé, sur l'arrière-fond d'autres coïncidences: les deux romans sont publiés la même année et ils sont l'œuvre d'écrivains qui, situant tous deux l'action à la campagne, doivent textualiser des personnages dont l'appartenance sociale et culturelle est assez différente de la leur; les deux romans s'inscrivent dans des traditions génériques ayant reçu la même dénomination dans leur lieu d'origine, manifestent un intérêt marqué pour la réalité sociale immédiate et travaillent des diégèses exhibant, malgré leurs différences de détail, une notable ressemblance structurale. Comment ne pas 
s'étonner alors de trouver dans Vidas secas cette remarque qu'on dirait sortie du discours du narrateur de Trente arpents après l'incendie de la grange des Moisan:

D'ordinaire, la famille parlait peu. Et après ce désastre-là, ils vivaient tous en silence, rarement ils laissaient échapper quelques mots courts ${ }^{6}$.

Plus que de ressemblance, on peut parler ici d'une identité propositionnelle devant laquelle d'aucuns se sentiraient justifiés d'employer un comparatisme intégrateur ${ }^{7}$ conduisant à l'identification d'une communauté idéologique entre les deux romans. Cette méthode s'avérerait toutefois inadéquate, parce qu'elle forcerait l'analyse à oublier que la signification des formulations des textes fictionnels est régie par une régularité tout à fait différente des régularités régissant la signification dans d'autres types de texte. Un roman peut être tenu - même si la comparaison est abusive parce qu'accordant à un simple ouvrage les propriétés de grandeurs bien plus vastes - pour une formation discursive à part entière. Chaque roman instaure en effet une régularité spécifique entre ses formulations, les subordonne à des régimes de représentation déterminés, les soumet à des modes d'énonciation particuliers, les rattache à des voix singulières, leur accorde un ton, des accents, des inflexions, les mesure à d'autres formulations venant de la même voix ou d'autres, les transforme en somme en énoncés qui ne se confondent pas avec leur simple contenu propositionnel. Par conséquent, les questions posées à des formulations similaires venant de deux romans différents doivent permettre d'établir si ces formulations accueillent le même énoncé ou bien si, au-delà de leur similitude, elles sont investies d'énoncés distincts. Pour être à même de formuler de telles questions, il faudra maintenant caractériser de manière succinte les modalités d'existence du silence humain dans le roman de Graciliano Ramos.

Vidas secas raconte quelques moments isolés de l'histoire d'une misérable famille paysanne - Sinha Vitoria, Fabiano, leurs deux enfants et la chienne Baleia - forcée par la sécheresse à parcourir les terres du Nord-est brésilien. à la recherche d'un lieu fertile. Le discours du narrateur attribue à Fabiano des traits qui le rapprochent d'Euchariste Moisan. Celui-ci admirait en particulier le discours du Français Albert Chabrol; Fabiano admirait les mots longs et difficiles des gens de la ville ${ }^{8}$. Euchariste se méfie de la labilité sémantique de ses paroles, après avoir vu à quel détournement de sens elles pouvaient être soumises par les avocats; quant à Fabiano: Parfois il disait une chose sans intention d'offenser, ils en entendaient une autre, et là commençaient les problèmes 9 . De là que Fabiano partage avec Euchariste la conviction que les gens lettrés se servent du langage dans des buts dolosifs: Chaque fois que les hommes instruits lui disaient des mots difficiles, il se faisait avoir. En les entendant, il sursautait. Évidemment, ils ne servaient qu'à cacher des escroqueries ${ }^{10}$. Fabiano partagera encore avec son lointain frère québécois le sentiment de l'inutilité du langage verbal pour communiquer avec les proches: Le seul être vivant qui le comprenait était sa femme. Il n'avait même pas besoin de parler : les gestes suffisaient ${ }^{11}$. 
Parfois, ce sont les comportements verbaux des protagonistes ou les situations de parole vécues par eux qui invitent au rapprochement: Euchariste sera hanté pour avoir dit des choses qui l'ont perdu - Pourquoi d'ailleurs avoir parlé? Une fois de plus les mots l'avaient trahi, les traîtres mots dont il avait l'habitude de se méfier ${ }^{12}$; Fabiano de même: Deux impies lui étaient tombé dessus, une barre de fer lui frappait la poitrine, une autre le dos, il s'était traîné comme un poulet mouillé. Tout ça, parce qu'il s'était échauffé et avait dit un mot inconsidérément ${ }^{13}$. Euchariste répétera certains mots à haute voix, dans l'espoir de les intégrer à son vocabulaire - il lui arrivait de se répéter à voix haute, comme pour s'exercer: "Ma terre... ma terre... ma terre... ${ }^{14}$; Fabiano de même: Dans des moments de folie, Fabiano [...] disait des mots difficiles, tronquant tout, et se convainquait qu'il s'améliorait ${ }^{15}$.

Il convient de souligner que, en dépit de ces coïncidences, les personnages de Vidas secas sont victimes d'un laconisme bien plus marqué que celui attribué aux Moisan et à leurs voisins par les interventions du narrateur de Trente arpents. En fait, leurs performances sonores peuvent à peine être tenues pour des performances verbales, se situant plutôt à un stade prélinguistique: la conversation entre Fabiano et Sinha Vitoria n'était pas proprement de la conversation ${ }^{16}$; elle, on l'entendra lancer son interjection gutturale $^{17}$, lui, parler un langage chanté, monosyllabique et guttural ${ }^{18}$. Un langage qui, comme tant d'autres traits du personnage, le placera moins du côté de l'humanité que de celui de l'animalité ${ }^{19}$ : Parfois, il employait dans sa relation avec les autres la même langue qu'il utilisait pour s'adresser aux bêtes - des exclamations, des onomatopées. En vérité, il parlait peu. Il admirait les mots longs et difficiles des gens de la ville, il tentait d'en reproduire quelques-uns, en vain, mais il savait qu'ils étaient inutiles et peut-être dangereux ${ }^{20}$.

Cette défiance du personnage face à la parole parle-t-elle, comme dans le cas de Trente arpents, d'une difficulté chez l'écrivain de relever le défi qui consiste à accorder la parole à ses héros? Cela ne fait pas de doute. Comme pour Ringuet, la distance qui sépare Ramos de ses personnages illettrés ne peut que comporter des obstacles au moment de textualiser leurs voix. Mais il ne fait pas de doute non plus que, à la différence de Ringuet, Ramos ne scotomise pas la difficulté, qu'il en reconnaît la force et s'y attaque, ce qui lui permet d'orienter ses influences, de limiter ses effets et de transformer ceci en attribut crédible de ses personnages. Un attribut qui, au contraire de ce qui arrive dans Trente arpents, n'est pas appliqué aux héros de manière sporadique - il est présent tout au long du roman, il n'entre pas en contradiction avec leurs comportements, et ne nécessite donc pas de théorie généralisatrice pour être justifié. La difficulté de parole des paysans de Ramos ne vient pas d'une "nature paysanne» quelconque mais de l'histoire de ces personnages; pleinement textualisée, elle est un des fils servant à tisser la vie fictionnelle de la famille de migrants. Ainsi, par exemple, le silence de Fabiano face aux questions de l'un de ses enfants reproduira un souvenir qui remonte à sa propre enfance: Il essaya de se rappeler le temps de son enfance, il s'est vu petit, malingre, la chemise sale et déchirée, accompagnant son père aux champs, l'interrogeant en vain ${ }^{2 !}$. 
Le roman lui-même est le produit d'une interrogation, celle à travers laquelle son narrateur accepte de refaire le cheminement de ses personnages, au risque, parfois, de recevoir le silence comme réponse. Cette possibilité, toujours présente, oblige le narrateur à se tenir près de ses personnages, à essayer de converser dans leur propre langue, même si elle n'a rien de cultivé, même si cela le contraint à laisser de côté le langage "littéraire".

Ce n'était pas proprement de la conversation: c'était des phrases isolées, espacées, avec des répétitions et des incongruités. [...] $/ \mathrm{ls}$ montraient les images qui leur venaient à l'esprit, et les images se succédaient, se déformaient, il n'y avait pas moyen de les dominer ${ }^{22}$.

Le passage ne sert pas qu'à caractériser les conversations des personnages. Il contient aussi une excellente description du roman lui-même, il définit le résultat d'une négociation. Le narrateur convient de se tenir près de la langue de ses héros, d'oublier que la tradition romanesque exige un savant dosage de portraits, pauses descriptives, narrations, dialogues, et mélange tout cela au risque de brouiller le discours global. Il consent à se servir le moins possible des opérateurs logiques, même si le résultat peut paraître incongru. Il accepte de se répéter, de ne pas tout savoir, de laisser des blancs, de confondre le passé et le présent, de se laisser porter par la force des épisodes, de ne pas introduire de liaison entre ces épisodes. Il opte pour parler lui aussi un langage onomatopéique en reproduisant le chapechape des espadrilles, pour ne pas signaler avec des italiques et des guillemets les mots de ses personnages, pour ne pas employer des termes qui ne puissent être dits dans la cantine où Fabiano est humilié par le soldat jaune, pour utiliser, sans les traduire, des vocables que le dictionnaire ignore parce que locaux, archaïques ou trop plébéiens.

Tout cela, j'entends déjà l'objection, c'est des conventions littéraires. Oui, ça l'est. Mais il n'empêche qu'il fallait les inventer, ces conventions, pour réduire enfin la distance incommensurable entre un docte narrateur et ses béotiens de personnages, observés tantôt avec amusement, tantôt avec paternalisme. Distance qui avait caractérisé pendant plus d'un siècle le roman latino-américain et qui, en 1938, caractérisait encore le roman québécois.

Le narrateur de Vidas secas n'est pas, il est vrai, moins omniscient que celui de Trente arpents, mais il n'abuse pas de ce privilège pour introduire des explications étrangères au savoir de Fabiano, Sinha Vitoria, les enfants, et même la chienne Baleia, des explications qui éclaireraient l'origine de la sécheresse, l'exploitation dont sont l'objet les personnages, la misère de ceuxci, leurs humiliations, leur faim, leur silence. Autodidacte cultivé, militant de la gauche, homme politique, Ramos ne manquait sûrement pas d'une panoplie de bonnes raisons qu'il aurait pu transmettre à son narrateur pour que celui-ci, à la manière du narrateur ringuétien, scandât son discours avec des éclaircissements ethnographiques, sociologiques ou linguistiques. Mais, dans ce cas, il aurait dû subir les rebuffades de ces paysans qui, ligotés, n'auraient eu que le choix de poser pour la carte postale mais qui, bouche cousue, ne lui auraient pas livré un millimètre carré de leur monde. Le narrateur de Vidas 
secas reconnaîtra d'emblée l'hermétisme de ses personnages et l'existence d'une distance entre son discours et le leur, et il acceptera en premier lieu de faire passer sa voix - la seule possible au début du texte - à travers leur regard:

Sur la plaine rougeâtre, les juazeiros étendaient deux taches vertes. Les malheureux avaient marché toute la journée, ils étaient fatigués et affamés. D'ordinaire, ils marchaient peu mais, s'étant assez reposés sur le sable de la rivière sèche, le voyage avança trois bonnes lieues. Cela faisait des heures qu'ils cherchaient une ombre. Le feuillage des juazeiros apparut au loin, à travers les branches pelées de la catinga clairsemée.

Le mouvement de va-et-vient du regard des personnages au savoir du narrateur annonce le type de partage auquel le texte donnera lieu. C'est en acceptant de parler depuis leur regard et en usant de ce langage dépouillé, fortement paratactique, privé de tout savoir étranger au savoir des personnages, que le narrateur arrivera à saisir des bribes de leur parole. L'omniscience du narrateur de Vidas secas est une omniscience pauvre ${ }^{23}$ et va de pair avec une pratique élocutive étrangère à l'omniloquence, pratique qui conduit de manière progressive à l'adoption d'un discours indirect libre favorisant l'émergence intermittente des discours intérieurs de Fabiano, Sinha Vitoria, les enfants et même de la chienne Baleia. Ce discours, comme il a été signalé par un critique ${ }^{24}$, permettra à la fois de lever la contradiction qui consiste à faire parler des personnages dont il est posé qu'il leur est difficile de parler et de figurer cette difficulté. Cela suppose chez le romancier une posture iconoclaste à l'égard du formalisme romanesque — au sens brechtien _, l'abandon de toute formule convenue pouvant empêcher le roman de parler comme ses personnages, la subordination du choix des procédés techniques à l'accomplissement du projet hylique ${ }^{25}$. Le roman sera fragmentaire comme la parole de ses personnages, il sera cette parole. C'est pourquoi on a pu affirmer que sa construction souffrait d'un défaut technique considérable, celui de l'éparpillement de ses parties: les treize tableaux que ses chapitres constitueraient ne s'articuleraient pas avec suffisamment de fermeté et d'assurance ${ }^{26}$. La remarque suppose une référence au roman comme forme canonique intouchable et passe à côté de ce qui participe à la valeur esthétique de Vidas secas: la ferme liaison entre sa forme délibérément déliée et le discours troué de ses personnages ${ }^{27}$. D'autre part, en nommant «tableaux » les différents fragments dans lesquels l'œuvre prend forme, la même remarque picturalise cette œuvre où, en vérité, le regard initial n'est là que pour permettre le passage à l'écoute à partir de laquelle se déploie toute la suite du roman ${ }^{28}$.

A l'exact opposé de Vidas secas, Trente arpents suit un itinéraire qui conduit de l'éphémère écoute initiale à un regard ne pouvant saisir les personnages que de l'extérieur. Quant à l'impossibilité absolue d'accomplir son projet hylique, elle n'est pas indépendante de sa soumission inconditionnelle à une forme romanesque toute faite et à un ensemble de techniques jamais mises en question, ni d'une soumission inconditionnelle de son narrateur à celui qu'il se donne comme narrataire. Cette soumission à une forme et à un 
narrataire, dont la nationalité - la même pour tous deux - est une donnée capitale, fait de Trente arpents le signe d'une situation de dépendance esthétique de type colonial. Une situation dans laquelle le romancier se conçoit comme pourvoyeur de matières premières autochtones - thèmes, motifs, personnages, objets, parlers, etc. -et opère avec la technologie qui lui vient de la métropole pour fabriquer des produits à être consommés par les métropolitains ${ }^{29}$.

Voilà la manière dont l'histoire passe au roman et dont le roman passe à l'histoire. Dans la mesure où l'on reconnaît que la fascination produite au Québec par la France est une donnée d'ordre sociologique, on peut avancer que l'échec de Trente arpents, partiellement déterminé par cette fascination, tient à une réalité sociale spécifique. On dira aussi que la dissemblance du succès de Trente arpents et de Vidas secas dans l'accomplissement de leur projet hylique et esthétique ne tient pas à une différence de talent entre deux écrivains mais aux différences entre deux situations historiques. Ou faudrait-il peut-être définir le talent non comme disposition naturelle ou individuelle mais comme aptitude façonnée par un environnement complexe, comme capacité déterminée par l'activité de l'écrivain dans une histoire littéraire spécifique et dans l'ensemble des relations qu'entretissent des forces diverses à un moment précis dans une société donnée. Dans ce cas, la dissimilitude entre les solutions techniques trouvées par Ramos et Ringuet pour résoudre des difficultés similaires ne sera pas qu'une différence entre deux savoir-faire; elle fera également signe à l'écart séparant deux conditionnements socioculturels de l'acte d'écriture et deux façons de répondre à ces conditionnements.

L'adoption par Ringuet d'une forme française ne tient pas qu'à un état de séduction collective. Dans la mesure où il apparaît historiquement que les littératures surgies outremer en prolongement des littératures européennes ont besoin de se référer à celles-ci tant qu'elles n'ont pas atteint leur autonomie, l'adoption d'une forme de l'ancienne métropole était pour lui inévitable. On ne doit pas oublier toutefois que la forme choisie n'est pas moins datée que le moment de l'écriture de Trente arpents et qu'entre les deux, il y a un siècle de distance. En fait, l'option de Ringuet pour des techniques propres au réalisme d'atmosphère est déterminée par l'absence derrière lui d'une tradition romanesque québécoise, par le manque de recherches esthétiques qui auraient pu permettre au roman québécois de se trouver une voie à soi. Cette absence, tributaire d'un isolement culturel décidé par les élites québécoises un siècle avant Trente arpents, se perpétuait en 1938, quand le réalisme balzacien était déjà suranné dans son lieu d'origine - entretemps, il y avait eu Dujardin, Gide, Proust... Tandis que Ringuet travaillait dans un environnement d'où le réalisme était absent ${ }^{30}$, Ramos ouvrait dans un espace culturel qui avait commencé à combattre le romantisme au XIX ${ }^{\mathrm{e}}$ siècle et qui, en 1922, année de l'explosion du modernisme brésilien, alors que la tradition du réalisme était déjà solide au Brésil, avait achevé de briser les liens de soumission culturelle qui unissaient le pays au Portugal et proclamé l'indépendance littéraire nationale. Une indépendance qui s'était manifestée dans le roman, entre autres, par le reniement du manichéisme langagier propre aux paradigmes littéraires 
précédents $^{31}$, par la fière adoption de la langue populaire et par une hybridation aiguë des langages sociaux. Une indépendance obligeant les écrivains à être des inventeurs.

Il est peut-être possible de discerner dans l'hybridation et la brésilianisation discursives pour lesquelles Ramos invente des formes nouvelles une autre manifestation de la participation de la littérature à l'histoire et de l'histoire à la littérature. Le premier de ces traits m'apparaît comme l'expression littéraire de l'intense métissage ethnique, linguistique et culturel de la société brésilienne - si différent de la ghettoïsation des cultures propre au Québec, où les termes de la diversité sont soumis à la juxtaposition et interdits de brassage. Quant à la textualisation de la langue populaire, elle parle d'un mode spécifique de présence de cette langue au sein de la société ainsi que du genre d'écoute dont elle fait l'objet de la part des travailleurs intellectuels. Trente arpents et Vidas secas parlent tous deux de l'étouffement dont la parole populaire est victime dans leurs sociétés de référence. Mais en outre, le fait que cette parole puisse prendre texte dans le roman de Ramos et que cette possibilité lui soit inaccessible dans le roman de Ringuet indique l'écart idéologique entre un romancier pour qui la parole populaire reste inaudible et un autre, sensible à chaque inflexion de cette parole. Plus encore, pour autant que les textes de Ringuet et de Ramos sont représentatifs du faire littéraire dans leurs espaces sociaux respectifs, l'écart séparant ces textes aux plans hylique et esthétique fait signe aux barrières qui, au Québec, s'interposent entre intellectuels et classes populaires et au fait qu'au Brésil, un grand nombre d'intellectuels a réussi à passer outre ces barrières. On me permettra ici de m'éloigner des textes (mais est-ce que je le fais vraiment?) pour rappeler que Graciliano Ramos était militant du Parti communiste brésilien. Aussi, dans la tendresse honteuse de Ringuet pour la parole de ses paysans, il y a peut-être une indication de la force avec laquelle, au Québec, ces chaires, tribunes et journaux dépositaires de l'éloquence nationale, dont Trente arpents parle en page 274, étouffent les voix populaires. En revanche, la promiscuité parolière de Ramos signale les trous qu'au Brésil les luttes populaires arrivent à percer dans l'artificielle homogénéité du réel créée par les discours des classes dirigeantes. Dans l'appareil intertextuel de Vidas secas (encore une fois, je ne m'éloigne pas' des textes) se font entendre les voix populaires ayant animé, au moment de l'écriture du roman, la colonne Prestes et l'Alliance nationale libératrice.

1. Cette section est le résumé d'un autre article qui, sous le titre «Le silence de Trente arpents", paraîtra dans un prochain numéro de Voix et images. Idéalement, l'article en question aurait dû paraître avant ce résumé, dont les formulations risquent d'avoir une allure trop péremptoire du fait que les démonstrations qui les justifient ont été ici omises. En attendant ces démonstrations, le lecteur pourra se référer à mon article "Pour une écoute bakhtinienne du roman latino-américain" (Études françaises. 20/1, Printemps 1984, p. 101-136), où j'identifie dans plusieurs textes des procédures que l'on retrouve dans Trente arpents. Il n'est pas sans intérêt de rappeler ici une curieuse situation. André Belleau prononça, à propos du texte de Ringuet, deux conférences. en mars et avril 1985 aux universités Laval et McGill respectivement (mention de la première m'a été faite bien plus tard par Michel van Schendel). Ces conférences, dont 
je connus le contenu une fois terminée la rédaction de «Le silence de Trente arpents», mettaient en lumière quelques-uns des aspects de la distribution de la parole dans Trente arpents que je croyais, les ayant trouvés bien avant dans des romans latinoaméricains, avoir été le premier à identifier chez Ringuet, au début de 1986, en écrivant mon texte sur son silence. Quand André Belleau et moi nous surprîmes mutuellement en nous communiquant les résultats coïncidents de nos enquêtes au plan technique du roman (il y avait aussi, bien sûr, et heureusement, plusieurs désaccords au plan de l'interprétation poétique et sociologique des données obtenues), nous coïncidâmes encore en attribuant la communauté de nos résultats à la leçon d'écoute que nous reçûmes de Mikhaïl Bakhtine. Inutile de dire combien il m'a été agréable que le hasard décide de me faire vivre, avec André Belleau, une aventure comparable, toutes proportions gardées, à celle de Edison et Cros (là aussi, il était question d'oreille), qui inventèrent le phonographe presque en même temps et sans savoir que l'autre était en train de le faire.

2. Gilles Marcotte, le Roman à l'imparfait, Montréal, Les éditions La Presse, 1976, p. 14.

3. Voir le strict partage de ces deux mots entre le narrateur et les personnages respectivement, dans l'épisode de la visite de Willie Daviau chez les Branchaud, p. 7I et suivantes (les références au foliotage renvoient à l'édition en livre de poche de J'ai lu, Paris, 1980).

4. Voir la scène mentionnée dans la note précédente, qui, parmi bien d'autres, montre à quel point, dans la réalité textuelle, les paysans ringuétiens sont loquaces, voire hâbleurs.

5. Op. cit., p. 8.

6. Editora Record, São Paulo, 1978. Les traductions des fragments de Vidas secas ont été faites par l'auteur de cet article.

7. Cf. Adrian Marino, «Le comparatisme des invariants: le cas des avant-gardes", Cahiers roumains d'études littéraires, No 1, 1976, p. 81-95.

8. Op. cit.. p. 21 .

9. Ibid., p. 104.

10. Ibid., p. 103

11. Ibid., p. 104.

12. Op. cit., p. 304.

13. Op. cit., p. 110.

14. Op. cit.. p. 37.

15. Op. cit., p. 26.

16. Ibid.. p. 21.

17. Ibid., p. 11.

18. Ihid., p. 21 .

19. Pour les enfants, les choses ne sont pas différentes: l'aîné. qui est capable de maintenir des conversations avec la chienne Baleia, avait un vocabulaire presque aussi pauvre que le perroquet mort au temps de la sécheresse (p. 59). Et ailleurs: Comme il ne savait pas bien parler, l'enfani balbutiait des expressions compliquées, répétait les syllabes, imitant les beuglements des animaux, le bruit du vent, le cri des cogs ( $\mathbf{p} .62$ ).

20. Ibid., p. 21.

21. Ibid., p. 42.

22. Ibid., p. 66

23. J'emprunte cet adjectif à Haroldo de Campos qui l'utilise pour caractériser le style du roman de Graciliano Ramos dans "Arte pobre, tempo de pobreza, poesia menos», Os pobres na literatura brasileira, Sâo Paulo, Editora brasiliense, 1983, p. 181-189.

24. R.A. Lawton, "Vidas sêcas: entre l'être et les choses", dans Séminaire Graciliano Ramos. Vidas sêcas, Poitiers, Centre de recherches latino-américaines, 1972, p. 55-84. 
25. Pour la notion de hylique, voir mes articles «Pour une écoute bakhtinienne du roman latino-américain", op. cit., "Cortazzar ou l'impossibilité du thrène". dans Liberté, no 153, Juin 1984, p. 94-108, et celui annoncé dans la note 1.

26. Cf. Alvaro Lins, "Valores e misérias das Vidas secas " (1947), texte publié en épilogue de l'édition utilisée par l'auteur de cet article.

27. Que signifie l'affirmation esthétique de l'unité de contenu et de forme dans une auvre bien faite sinon que le même diagramme structural régit ses différents niveaux d'organisation? Umberto Eco, La struttura assente, Milan, Bompiani, 1968, p. 156.

28. Pour R.A. Lawton, l'action de Vidas secas est un agi parlé: l'action, toute l'action, consiste à parler. Op. cit., p. 82.

29. Il n'est pas inutile de transcrire ici une nouvelle qui rappelle que les traditions se perpétuent et que cette perpétuation est encouragée par les instances de consécration et de légitimation de ce qui, pour une bonne partie de la production esthétique québécoise. continue de tenir lieu de métropole:

PARIS (PC) - L'écrivain québécois Michel Tremblay a été choisi, hier, lauréat du Prix Québec-Paris 1984 parce que, entre autres raisons, a expliqué le président du jury, «il a su bien gérer le joual». Pour certains.

il y en a trop, a poursuivi le poète et critique littéraire Alain Bosquet, qui préside le jury de 10 membres. Pour d'autres, au contraire, il y a une économie très bien gérée entre les dialogues en joual pur - si j'ose dire, ou il faut dire impur? - et le langage du récit qui, lui, peut être dans un français impeccable. Le Devoir, 6 février 1985.

30. Il y avait eu, il est vrai, la Scouine (1918), texte avec lequel Albert Laberge était d’ailleurs allé bien plus loin sur le plan de la recherche hylique et esthétique que Ringuet avec le sien. Mais un seul roman, condamné de surcroît au silence, ne peut constituer une tradition réaliste.

31. Mais si les naturels de cette terre se servent d'une langue aussi méprisable dans leur conversation - lit-on dans Macunaima (1928), de Mário de Andrade - il leur suffit de prendre la plume pour se dépouiller de tant d'âpreté et alors surgit l'Homme Latin de Linné, qui s'exprime dans un autre langage, très proche du virgilien, au dire d'un panég.vriste, doux idiome qui, avec une impérissable élégance, s'intitule: langue de Camöes! (traduit de l'édition de Livraria Martins, São Paulo, 1979, p. 107). Macunaima, bien sûr, va à l'encontre de ce manichéisme.

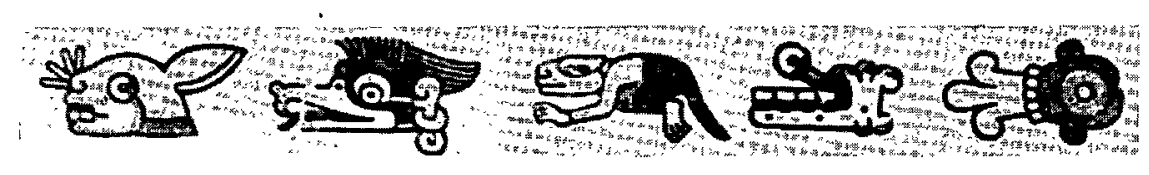

